\title{
Article
}

\section{Split-belt adaptation and gait symmetry in transtibial amputees walking with a hybrid EMG controlled ankle-foot prosthesis}

Kannape, Oliver and Herr, HM

Available at http://clok.uclan.ac.uk/15278/

Kannape, Oliver ORCID: 0000-0002-2930-4118 and Herr, HM (2016) Split-belt adaptation and gait symmetry in transtibial amputees walking with a hybrid EMG controlled ankle-foot prosthesis. 2016 38th Annual International

Conference of the IEEE Engineering in Medicine and Biology Society (EMBC) .

It is advisable to refer to the publisher's version if you intend to cite from the work. http://dx.doi.org/10.1109/EMBC.2016.7591964

For more information about UCLan's research in this area go to http://www.uclan.ac.uk/researchgroups/ and search for <name of research Group>.

For information about Research generally at UCLan please go to http://www.uclan.ac.uk/research/

All outputs in CLoK are protected by Intellectual Property Rights law, including Copyright law. Copyright, IPR and Moral Rights for the works on this site are retained by the individual authors and/or other copyright owners. Terms and conditions for use of this material are defined in the policies page. 


\title{
Split-belt adaptation and gait symmetry in transtibial amputees walking with a hybrid EMG controlled ankle-foot prosthesis
}

\author{
Oliver A. Kannape, Member, IEEE, Hugh M. Herr, Member, IEEE
}

\begin{abstract}
Our ability to automatically adapt our walking pattern to the demands of our environment is central to maintaining a steady gait. Accordingly, a large effort is being made to extend and integrate this adaptability to lower-limb prostheses. To date, the main focus of this research has been on short term adaptation, such as in response to a terrain transition or a sudden change in the environment. However, long term adaptation and underlying sensorimotor learning processes are critical to optimizing walking patterns and predictively changing our gait when faced with continued perturbations. Furthermore, investigating these processes in lower-limb amputees may provide a unique window into the interplay between sensory driven adaptation and top-down cerebellar modulation of locomotor reflexes and may potentially help alleviate gait asymmetries. In the current exploratory study, we therefore investigated adaptation, sensorimotor learning, and gait symmetry in a group of transtibial amputees walking with a hybrid-EMG controlled powered prosthesis and matched controls (both groups $\mathrm{N}=3$ ). Participants were asked to perform a split-belt walking trial during which the belt on the affected side ran at twice the speed of the contralateral belt $(1.0 \mathrm{~m} / \mathrm{s}$ and $0.5 \mathrm{~m} / \mathrm{s}$ respectively). Adaptation, sensorimotor learning, and symmetry are compared to two baseline conditions. Initial results illustrate that the amputees were readily able to use the hybrid controller, modulated their EMG depending on treadmill speed, and successfully adapted their gait during split-belt walking. However, the temporal gait parameters suggest that amputees used a different adaptation technique and showed reduced sensorimotor learning, while gait symmetry was improved, in the short term, post-adaptation.
\end{abstract}

\section{INTRODUCTION}

Human locomotion is highly adaptable and automatically changes in response to the demands of the walking terrain or context [1]. In the short term, this adaptability is crucial for maintaining balance and quickly reacting to unpredictable perturbations. In the long term, adaptation and with it sensorimotor learning are further important to optimize gait and adjust walking patterns predictively, when faced with persistent perturbations [2]. Crucially, gait adaptability is also one of the major challenges in the development of controllers for active lower-limb prostheses. Current efforts are largely focused on short term adaptation, i.e. safely modifying prosthesis behavior to surface transitions from e.g. level ground to stair ambulation. These controllers range from finite

*Research supported by the Department of Defense award number 6920559 and 6920877. Oliver A. Kannape was further supported by the Swiss National Science Foundation grant PBELP3 140656.

Oliver A. Kannape was with the Center for Extreme Bionics, MIT Media Lab, Massachusetts Institute of Technology, Cambridge, MA 02139 USA. state machines that are intrinsically driven using on-board sensors [3], [4] to direct proportional myoelectric control [5], as well as hybrid designs [6]-[8]. However, none of these prostheses or interfaces have thus far been used to investigate long term locomotor adaptation. Importantly, the underlying sensorimotor learning mechanisms may prove key to optimizing prosthesis control by utilizing the interaction between sensory driven adaptation and top-down (cerebellar) modulation of the involved spinal reflexes [9]. While initial studies illustrate the role of afferent, proprioceptive input on gait adaptation [10], to date, no comparable amputee-data have been published. Furthermore, clinical research point to a potential benefit of running locomotor adaptation trials as a rehabilitation measure as participants' post-adaptation gait symmetry improved compared to baseline performance [11]. Gait asymmetries have been linked to the development of knee osteoarthritis of the leading limb, a common secondary impairment in lower extremity amputees [12].

The aim of the current study was therefore to investigate sensorimotor learning during gait adaptation in transtibial amputees, walking with a hybrid EMG controller, and its effects on post-adaptation gait symmetry. Gait adaptation was imposed by asking participants to walk on a split-belt treadmill, with the belt on the affected side running at twice the speed of the contralateral belt. Extending our previous work on the hybrid EMG controller [6], [8] we hypothesized that transtibial amputees would be able to i) adjust their residual limb muscle EMG according to treadmill velocity and ii) adapt to the split-belt walking condition by normalizing temporal gait characteristics over the 10minute adaptation trial, but iii) show reduced sensorimotor learning as evidenced by the wash-out phase immediately following the adaptation trial. Finally, we compared amputee gait symmetry pre- and post-adaptation.

\section{MethodS}

\section{A. Prosthesis and Hybrid Controller}

1) BiOM Ankle-Foot Prosthesis

The BiOM ankle-foot prosthesis used for this study was developed by BionX and is a successor to the series of prototypes developed in the Biomechatronics Group of the MIT Media Laboratory [13], [14]. It is a self-contained device having the mass and size of the intact biological ankle-foot

He is now with the School of Psychology, University of Central Lancashire, Preston, PR12HE UK (email: okannape@uclan.ac.uk).

Hugh M. Herr is with the Center for Extreme Bionics, MIT Media Lab, Massachusetts Institute of Technology, Cambridge, MA 02139 USA (e-mail: hherr@mit.edu). 
complex. The basic architecture of the electromechanical design consists of a unidirectional spring in parallel to serieselastics actuator. The prosthesis is capable of varying impedance during stance, providing net positive mechanical energy during push-off and position control during swing.

\section{2) Ankle Push-off and Proportional Myoelectric control}

Nearly $80 \%$ of the mechanical work required to complete each gait cycle is generated by the gastrocnemius muscle in late-stance [15], [16]. This behavior is emulated by the prosthesis' onboard control system as it adapts the push-off, provided before toe-off, to the participants' walking characteristics. An increase in the sensed prosthetic ankle joint torque triggers an increase in the torque generated by the actuator during mid- to late stance phase, resulting in an increase in net positive ankle work production. The onboard controller applies a velocity-dependent gain to generate the output torque. As described in [8] when proportional myoelectric control is used, this gain component is linearly dependent on the EMG signal (cf. below for detailed thresholding procedure). To avoid an undesired drop in torque during ankle push-off, due to e.g. muscle fatigue or a fault in EMG signal, the gain term is prevented from decreasing in this phase of each gait-cycle and can only increase with increasing muscle recruitment or remain stable until toe-off.

\section{B. EMG Unit}

\section{1) Measurement Module}

The EMG measurement module consists of a single board computer (Version 2, Raspberry Pi Foundation, Cambridgeshire, UK), a 16-channel analog signal amplifier (RHA2216, Intan Technologies, Los Angeles, CA) and a 16bit analog to digital Converter (AD7980, Analog Devices, Norwood, MA). EMG signal is recorded using Philips SmartTrace electrodes (Philips, Amsterdam, Netherlands), which are placed above the gastrocnemius and tibialis anterior muscles and patella (reference electrode) of the residual limb, inside the participant's liner.

\section{2) Signal Processing}

The EMG signal was sampled at $1 \mathrm{kHz}$ and high-pass filtered using a $4^{\text {th }}$ order Butterworth filter with a cutoff frequency of $80 \mathrm{~Hz}$ to eliminate the main motion artifacts. It was subsequently rectified before being averaged over a moving window of $150 \mathrm{~ms}$. This average value was then used to scale the EMG signal to a percentage of the maximum gain set during tuning of the BiOM. The scaled EMG value was sent to the $\mathrm{BiOM}$ at a frequency of $125 \mathrm{~Hz}$ and used solely to control ankle push-off.

\section{3) Tuning and Threshold Detection}

At the beginning of the study a prosthetist tuned the BiOM for the participant. The torque gain was set to normalize the work performed by the prosthesis. An EMG threshold value was then determined to distinguish between an intentional activation of the gastrocnemius muscle and baseline noise due to motion artifacts and involuntary muscle activation. This was achieved by recording 10 steps with no voluntary muscle activation, followed by ten steps with close to maximal muscle activation. The thresholds were then manually set so
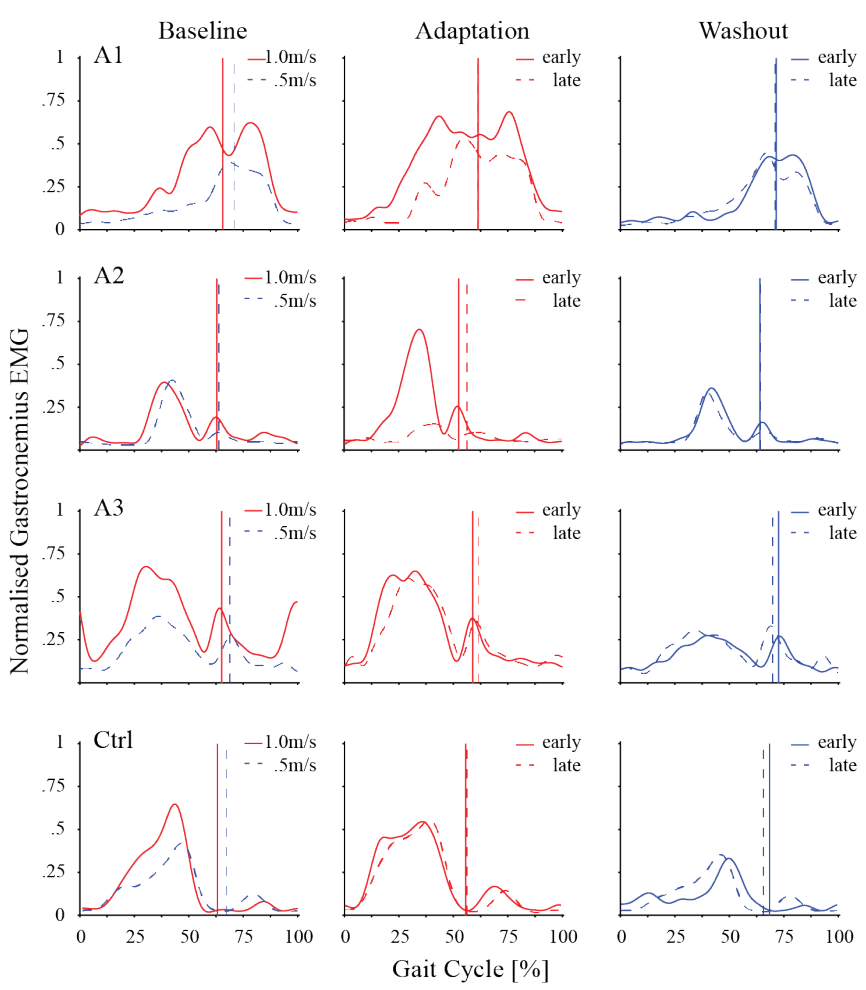

Figure 1 Gastrocnemius EMG. Profiles for the three amputees (affected side) and the average for the control participants are shown (matched side); vertical lines indicate toe-off. Each profile represents an average of 20 trials. All amputees were able to recruit their residual limb muscle to scale ankle push-off of the prosthesis.

that there was no interference due to involuntary muscle activation. The EMG scale remained $0 \%$ until the lower threshold was surpassed and then linearly depended on the EMG amplitude with an EMG scale of $65 \%$ corresponding to the manually tuned torque gain. This significantly increased the torque range available to the participants as they could disable the output torque of the BiOM entirely, such as may be desired during stair descent, or increase the gain beyond the maximal gain set for intrinsic control. Furthermore, an initial bias of $30 \%$ was introduced once EMG surpasses the lower threshold.

\section{Experimental Procedure}

Participants were asked to briefly walk on level ground using the hybrid interface and then step on the instrumented treadmill. They then completed four walking trials:

1) Baseline Fast

Both belts are set to $1.0 \mathrm{~m} / \mathrm{s}$; the trial lasts 1 minute.

2) Baseline Slow

Both belts are set to $0.5 \mathrm{~m} / \mathrm{s}$; the trial lasts 1 minute.

3) Adaptation

Belt one, on the participant's affected side is set to $1.0 \mathrm{~m} / \mathrm{s}$; belt two is set to $0.5 \mathrm{~m} / \mathrm{s}$; the trial lasts 10 minutes.

\section{4) Washout / Post-Adaptation}

Both belts are set to $0.5 \mathrm{~m} / \mathrm{s}$, as in the Baseline Slow condition; the trial lasts 5 minutes.

\section{Participants}

Three transtibial amputees (height: $180 \mathrm{~cm} \pm 10$, weight: 

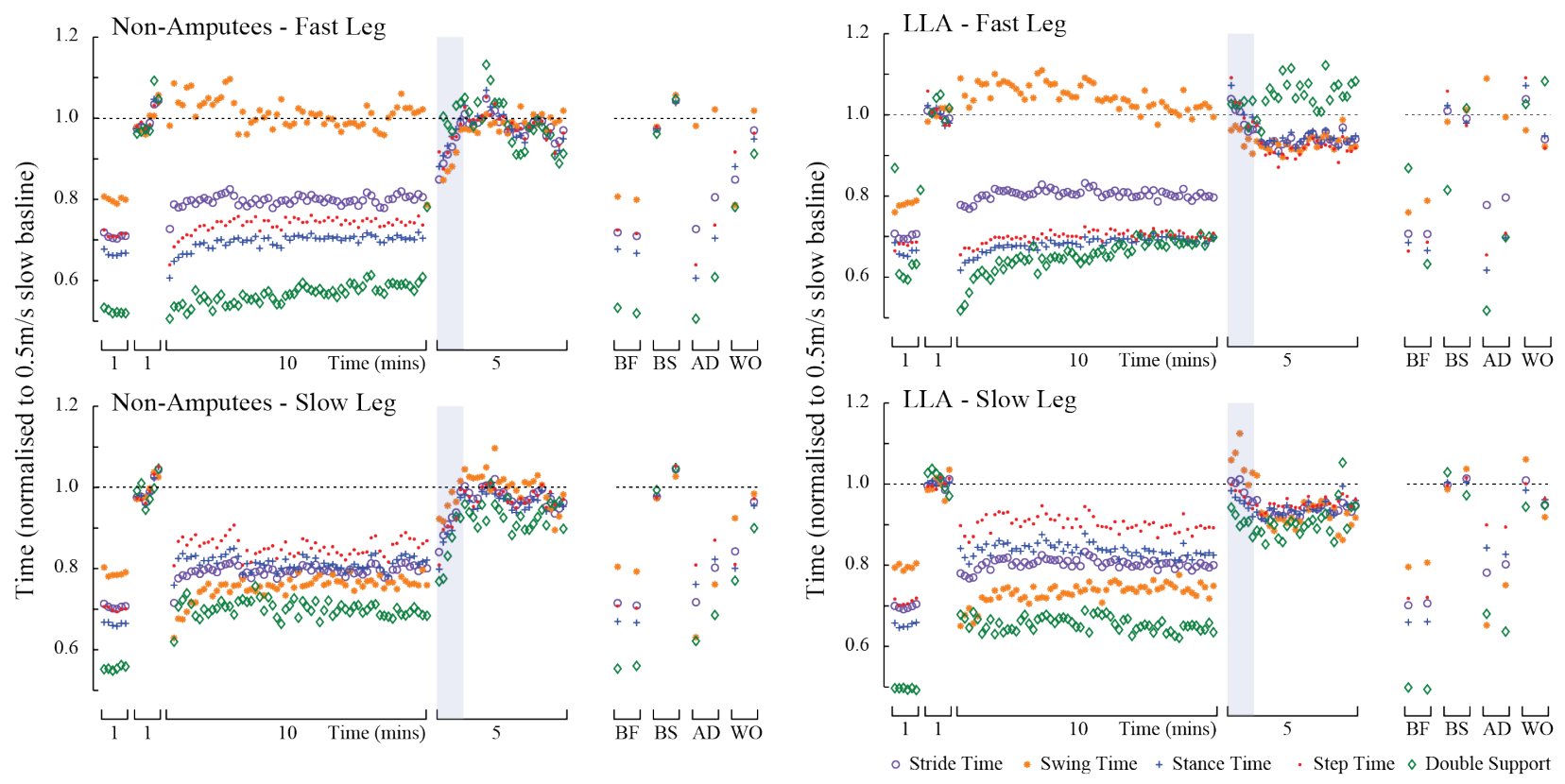

Figure 2 Temporal Gait Parameters. Stride-, swing-, stance-, and step time, as well as double support time are shown for both control participants and amputees. These are averaged in 10 second bins and divided into the different conditions: baseline fast (1 min at $1.0 \mathrm{~m} / \mathrm{s})$, baseline slow (1min at $0.5 \mathrm{~m} / \mathrm{s})$, adaptation (10mins), washout (5mins at $0.5 \mathrm{~m} / \mathrm{s}$ ). All parameters are normalized to the baseline slow condition. Initial results illustrate that the amputees (LLA) were able to adapt to the split-belt condition. The relative changes in step time/swing time compared to non-amputees suggest that this may be due to a different compensatory strategy, with more time spent on the slow, unaffected limb. Furthermore, the washout of the learnt motor program that is visible for the non-amputee participants is not evident for the LLA (highlighted by the shaded bars). The change in double support time in LLA post-adaptation is indicative of an increased gait symmetry and points to a potential training benefit of split-belt walking.

$79 \mathrm{~kg} \pm 9$ ) as well as three matched non-amputees (height: $179 \mathrm{~cm} \pm 13$, weight: $73 \mathrm{~kg} \pm 8$, dominant hand/foot corresponding to amputee's affected side) participated in the study. All amputees commonly wear conventional energy storage and return prosthesis but are experienced $\mathrm{BiOM}$ users. Participant A2 is a congenital amputee. The study was approved by MITs Committee on the Use of Humans as Experimental Subjects (COUHES) and the participant gave informed consent prior to commencing the study.

\section{E. Data Analysis and Processing}

Kinematic, kinetic, and EMG data were simultaneously collected. To measure kinematic and kinetic data, participants walked on a split-belt instrumented treadmill (Bertec, Columbus, OH, USA) within a motion capture space. Each tread independently measured shear and vertical forces at a sampling rate of $1000 \mathrm{~Hz}$. Reflective markers $(15 \mathrm{~mm}$ diameter) were placed on the participant's body at 46 locations, and their three-dimensional locations were measured at a sampling rate of $100 \mathrm{~Hz}$ by 12 infrared cameras (model: T40s, Vicon Motion Systems Ltd, Oxford, UK). The locations of these markers followed the Helen Hayes marker model, and were chosen to track joint motion. Lower limb EMG was collected from the lateral head of the medial gastrocnemius, tibialis anterior, rectus femoris, and biceps femoris, bilaterally. Residual limb EMG was recorded as described under section II. B. All other muscle groups were recorded using the Delsys Trigno Wireless EMG system (Delsys, Natick, MA, USA) with a $1 \mathrm{kHz}$ sampling rate.

Heel strikes were determined using the vertical force on the treadmill when in excess of $0.5 \mathrm{~N}$ after a prolonged period of no force, corresponding with a swing phase. Each trial was divided into individual gait cycles that began and ended with heel strike of the same foot, and then normalized in time by percent gait cycle. Sensor data from the BiOM and EMG data from the single board computer were recorded separately but synchronized using a $1 \mathrm{~Hz}$ trigger logged to the BiOM's data stream. This in turn was synchronized by matching the heelstrike data from the BiOM with that of the Bertec treadmill.

\section{RESULTS AND DISCUSSION}

\section{A. Baseline Walking}

As illustrated in Figure 1, all amputees were able to recruit their muscle and thereby control the torque generated by the prosthesis before ankle push-off. Peak EMG and toe-off shifted forwards when walking at $1.0 \mathrm{~m} / \mathrm{s}$ (toe-off: $64.85 \% \mathrm{GC} \pm 1.53)$ compared to $0.5 \mathrm{~m} / \mathrm{s}(67.92 \% \mathrm{GC} \pm 3.62)$. The amputee participants reported that it was significantly harder to time muscle contraction in the slow baseline condition whereas it seemed natural when walking at $1.0 \mathrm{~m} / \mathrm{s}$. The temporal parameters in Figure 2 further suggest larger gait variability in the slow walking condition in both groups.

\section{B. Adaptation}

In-line with the literature [17], gastrocnemius activity in the early adaptation trials increased in both length and amplitude compared to the fast baseline, also at $1.0 \mathrm{~m} / \mathrm{s}$. This level of activation remains fairly constant over the 10 -minute period, as observed for A3 and the control participants. Participants A1 and in particular A2 reported muscle fatigue as the trial went on, as evidenced by their respective EMG profiles (Figure 1). Fast or reactive adaptation is clearly illustrated in 
both amputees and non-amputees as stance times for the slow leg increase immediately whereas stance times for the fast leg decrease, see Figure 2; this appears to be pronounced in the amputees. Slow adapting changes are also apparent in both groups. Double support time is initially strongly asymmetrical before gradually normalizing to the level of the slow leg over the length of the 10-minute trial.

\section{Post-Adaptation and Washout}

Sensorimotor learning is illustrated by an initial continuation of the asymmetric gait acquired in the split-belt condition [17]. Conversely, if no learning occurred, participants should immediately return to baseline gait characteristics. The so-called washout effect is clearly visible in the temporal parameters of the non-amputee participants (cf. Figure 2, shaded areas). It takes approximately one minute for all parameters to return to the baseline performance at $0.5 \mathrm{~m} / \mathrm{s}$. This washout is not as evident for the amputees suggesting that learning of the new motor program may not have been as deep and resembles previously described limited learning in nonamputees walking with altered proprioceptive feedback [10].

\section{Gait Symmetry}

Previous literature in clinical populations has pointed to the potential benefits of split-belt treadmill walking for improving gait symmetry [11]. Our initial results indicate that this may also apply to lower-limb amputees as illustrated by the change in double support time post-adaptation, Figure 2. A small asymmetry of approximately $35 \mathrm{~ms}$ was apparent in the initial baseline condition at $0.5 \mathrm{~m} / \mathrm{s}$ but essentially vanishes $(<1 \mathrm{~ms})$ post-adaptation. This appears reversed in Figure 2 due to the baseline normalization. Of course these results are still preliminary and should be treated with caution due to the limited sample size. A further concern is the slow baseline speed, far below the participants' preferred walking speed, and itself linked to increased gait variability.

\section{CONCLUSION}

In conclusion, our results illustrate that the transtibial amputees were able to use the hybrid controller for the powered ankle-foot prosthesis during treadmill walking by modulating their EMG power. As hypothesized, all participants adapted to the split-belt condition by adjusting the spatiotemporal parameters of their gait. In the nonamputees this resulted in clear motor learning, as evidenced by the initial continuation of the asymmetric gait postadaptation. Further in-line with our hypotheses, our data suggest reduced sensorimotor learning in lower-limb amputees and hint at a compensatory adaptation technique. In combination with the reported improved gait symmetry in lower limb amputees post-adaptation, our findings highlight the importance of research into long term adaptation in lowerlimb amputees both to optimize biomimicity of prosthesis control but also to explore the use of split-belt training as a rehabilitative measure.

\section{REFERENCES}

[1] J. T. Choi and A. J. Bastian, "Adaptation reveals independent control networks for human walking," Nat Neurosci, vol. 10, pp. 1055-62, Aug. 2007.
[2] K. G. Pearson, "Neural adaptation in the generation of rhythmic behavior," Annu Rev Physiol, vol. 62, pp. 723-53, 2000.

[3] H. M. Herr and A. M. Grabowski, "Bionic ankle-foot prosthesis normalizes walking gait for persons with leg amputation," Proc. Biol. Sci., vol. 279, no. 1728, pp. 457-464, Feb. 2012.

[4] J. Markowitz, P. Krishnaswamy, M. F. Eilenberg, K. Endo, C. Barnhart, and H. Herr, "Speed adaptation in a powered transtibial prosthesis controlled with a neuromuscular model," Philos. Trans. $R$. Soc. Lond. B. Biol. Sci., vol. 366, no. 1570, pp. 1621-1631, May 2011.

[5] S. Huang, J. Wensman, and D. Ferris, "Locomotor Adaptation by Transtibial Amputees Walking with an Experimental Powered Prosthesis Under Continuous Myoelectric Control," IEEE Trans. Neural Syst. Rehabil. Eng. Publ. IEEE Eng. Med. Biol. Soc., Jun. 2015.

[6] J. Wang, O. A. Kannape, and H. M. Herr, "Proportional EMG control of ankle plantar flexion in a powered transtibial prosthesis," IEEE Int. Conf. Rehabil. Robot. Proc., vol. 2013, p. 6650391, Jun. 2013.

[7] L. J. Hargrove, A. M. Simon, A. J. Young, R. D. Lipschutz, S. B. Finucane, D. G. Smith, and T. A. Kuiken, "Robotic leg control with EMG decoding in an amputee with nerve transfers," N. Engl. J. Med., vol. 369 , no. 13, pp. 1237-1242, Sep. 2013.

[8] O. A. Kannape and H. M. Herr, "Volitional control of ankle plantar flexion in a powered transtibial prosthesis during stair-ambulation," Conf. Proc. Annu. Int. Conf. IEEE Eng. Med. Biol. Soc. IEEE Eng. Med. Biol. Soc. Annu. Conf., vol. 2014, pp. 1662-1665, 2014.

[9] D. S. Reisman, A. J. Bastian, and S. M. Morton, "Neurophysiologic and rehabilitation insights from the split-belt and other locomotor adaptation paradigms," Phys Ther, vol. 90, pp. 187-95, Feb. 2010.

[10] L. Jensen, T. Prokop, and V. Dietz, "Adaptational effects during human split-belt walking: influence of afferent input," Exp Brain Res, vol. 118, pp. 126-30, Jan. 1998.

[11] D. S. Reisman, R. Wityk, K. Silver, and A. J. Bastian, "Locomotor adaptation on a split-belt treadmill can improve walking symmetry post-stroke," Brain J. Neurol., vol. 130, no. Pt 7, pp. 1861-1872, Jul. 2007.

[12] D. C. Norvell, J. M. Czerniecki, G. E. Reiber, C. Maynard, J. A. Pecoraro, and N. S. Weiss, "The prevalence of knee pain and symptomatic knee osteoarthritis among veteran traumatic amputees and nonamputees," Arch. Phys. Med. Rehabil., vol. 86, no. 3, pp. 487-493, Mar. 2005.

[13] S. K. Au, H. Herr, J. Weber, and E. C. Martinez-Villalpando, "Powered ankle-foot prosthesis for the improvement of amputee ambulation," Conf. Proc. Annu. Int. Conf. IEEE Eng. Med. Biol. Soc. IEEE Eng. Med. Biol. Soc. Annu. Conf., vol. 2007, pp. 3020-3026, 2007

[14] S. K. Au, J. Weber, and H. Herr, "Powered Ankle-foot Prosthesis Improves Walking Metabolic Economy," Trans Rob, vol. 25, no. 1, pp. 51-66, Feb. 2009.

[15] D. A. Winter, "Energy generation and absorption at the ankle and knee during fast, natural, and slow cadences," Clin. Orthop., no. 175, pp. 147-154, May 1983.

[16] C. H. Soo and J. M. Donelan, "Mechanics and energetics of step-tostep transitions isolated from human walking," J. Exp. Biol., vol. 213, no. Pt 24, pp. 4265-4271, Dec. 2010.

[17] T. Ogawa, N. Kawashima, T. Ogata, and K. Nakazawa, "Predictive control of ankle stiffness at heel contact is a key element of locomotor adaptation during split-belt treadmill walking in humans," $J$. Neurophysiol., vol. 111, no. 4, pp. 722-732, Feb. 2014. 